\title{
Molecular targeted therapies for cancer: Sorafenib mono- therapy and its combination with other therapies (Review)
}

\author{
NUHA IBRAHIM ${ }^{1}$, YAN YU ${ }^{1}$, WILLIAM R. WALSH ${ }^{1}$ and JIA-LIN YANG ${ }^{2}$ \\ ${ }^{1}$ Surgical and Orthopaedic Research Laboratories, Prince of Wales Clinical School and \\ ${ }^{2}$ Sarcoma Research Group, Adult Cancer Program, Prince of Wales Clinical School and Lowy Cancer \\ Research Centre, Faculty of Medicine, University of New South Wales, Sydney, NSW 2052, Australia
}

Received November 24, 2011; Accepted December 29, 2011

DOI: $10.3892 /$ or.2012.1675

\begin{abstract}
Sorafenib is an oral multikinase inhibitor that acts by inhibiting tumor growth and disrupting tumor microvasculature through antiproliferative, anti-angiogenic and proapoptotic effects. It exerts these effects via inhibition of multiple targets including Raf serine/threonine kinases, vascular endothelial growth factor receptor tyrosine kinases; VEGFR-1, VEGFR-2, VEGFR-3 and platelet-derived growth factor receptor $\beta$ (PDGFR- $\beta$ ). Current literature shows that the deregulated signaling through these receptors is commonly seen in human tumors. In addition, sorafenib is also shown to induce apoptosis through downregulation of Mcl-1 in many cancer types. Hence, sorafenib as a single agent has shown promising activity in some cancers such as renal cell carcinoma (RCC), hepatocellular carcinoma (HCC) and thyroid cancers. Currently, the drug holds FDA approval for the treatment of advanced RCC and unresectable HCC. However, many clinical studies have indicated several limitations to the application of sorafenib as a single agent in various other cancers. Owing to these reasons and the potential of sorafenib to synergize with other anticancer therapies, its combination with other targeted agents and chemotherapy has been widely explored with promising results. In addition, it has also shown synergistic results when combined with radiation. This review summarizes the current basic and clinical studies on the effects and mechanisms of sorafenib either administered alone or in combination with other anticancer treatments.
\end{abstract}

\section{Contents}

1. Introduction

2. Sorafenib targets

Correspondence to: Dr Jia-Lin Yang, Level 2, Lowy Cancer Research Centre, Faculty of Medicine, University of New South Wales, Sydney, NSW 2052, Australia

E-mail: j.yang@unsw.edu.au

Key words: sorafenib, cancer, review, targeted therapy, combination therapy
3. Expression of sorafenib targets and mechanisms of action in cancers

4. Sorafenib monotherapy

5. Sorafenib combination therapy

6. Conclusions

\section{Introduction}

Since the 1980s, the field of cancer treatment has experienced a major paradigm shift from broad-spectrum cytotoxic chemotherapeutics to the development of targeted therapies tailored to inhibit cancer-specific pathways (1). This change was prompted by the limitations of the treatment mainstays in those days, which were surgery, chemotherapy and radiotherapy. Surgery was limited to early non-metastatic diseases, however, research demonstrates that solid tumors are frequently metastatic at presentation (2). Radiation and chemotherapy has limited capacity to discriminate between cancerous and normal cells and hence, results in severe side effects (3). Furthermore, solid tumors are inherently resistant to both radiation and chemotherapy. Therefore, the rationale for development of molecular targeted therapies was to overcome drug resistance, to make malignant cells more susceptible to suppression and damage whilst avoiding substantial toxicity to the rest of the body tissues and also to provide a higher therapeutic index.

The molecular targeted therapies include monoclonal antibodies, tyrosine kinase inhibitors, rapamycin pathway inhibitors, proteasome inhibitors and inhibitors of Raf kinase (4). Many of these drugs have shown therapeutic benefit in various cancers, however, several challenges are yet to be conquered by the developers. These include overcoming certain amount of acquired resistance in cancer cells, selecting suitable dosage schedules, determining the stage to start treatment and identifying relevant combination regimens. In addition, there appears to be a weakness in identifying the most important targets in cancer, as well as an inability to design drugs that would specifically confront the selected targets (4). Hence, the utility of successful targeted therapies is yet to be attained in many cancer patients.

Since the implication of deregulated Raf kinases in tumorigenesis and progression in many solid tumors, previous 
studies aimed at identifying a drug that will interfere with these targets. This led to the discovery of sorafenib in the late 1990s (1). Sorafenib acts on both the tumor and its microvasculature via multiple targets (5). This explains its broad activity in many tumor types and also its clinical effect in renal cell carcinoma (RCC) and hepatocellular carcinoma (HCC). In spite of this, sorafenib monotherapy was shown to be less successful in certain cancers such as sarcomas and melanomas due to reasons such as patient insensitivity and drug resistance. However, the multiple targets of sorafenib make it an attractive choice for combination therapy. Therefore, the combination of sorafenib with other drugs has been extensively investigated, indicating clinical benefits in many cancer types (6).

This review aims to explore: i) sorafenib targets and mechanisms of inhibition in several cancers, ii) sorafenib monotherapy and iii) combination therapy with chemotherapy, radiotherapy and other targeted agents.

\section{Sorafenib targets}

Sorafenib is a dual action multikinase inhibitor that targets both tumor cells and cells of the tumor vasculature. It inhibits tumor proliferation by potently inhibiting the Raf serine/ threonine kinases: Raf-1, wild-type B-Raf and oncogenic B-Raf V600E kinases in the MAPK pathway (7) (Fig. 1). These kinases are pivotal regulators of cellular proliferation and survival and in addition Raf-1 also interacts directly with anti-apoptotic and apoptotic regulatory proteins to prolong cell survival (1). Sorafenib has shown dose-dependent inhibition of tumor proliferation in various human cancer cell lines containing oncogenic K-Ras or B-Raf mutations, including breast, colon and pancreatic cancers (5).

Sorafenib also potently inhibits proangiogenic vascular endothelial growth factor receptor tyrosine kinases; VEGFR-1, VEGFR-2, VEGFR-3 and the platelet-derived growth factor receptor $\beta$ (PDGFR- $\beta$ ) (5). These receptor tyrosine kinases (RTKs) utilize the MAPK pathway to induce proliferation and prolong the survival of vascular endothelial cells, which gives rise to new blood vessels (2). Also they promote the proliferation, survival and recruitment of pericytes, which stabilize the newly formed blood vessels.

In addition, sorafenib has been shown to induce apoptosis in several tumor cell lines (5). Although this mechanism is not fully understood, it is commonly observed that sorafenib inhibits phosphorylation of the initiation factor eIF4E resulting in loss of the anti-apoptotic protein: myeloid cell leukemia-1 (Mcl-1). The downregulation of Mcl-1 by sorafenib causes the release of cytochrome $\mathrm{c}$ from mitochondria into the cytoplasm. This activates caspase and induces apoptotic cell death.

\section{Expression of sorafenib targets and mechanisms of action in cancers}

Research has shown that in about $30 \%$ of human cancers, signaling through the Raf kinase isoforms is deregulated (1). Activating oncogenic mutations in B-Raf, such as B-Raf $\mathrm{V} 600 \mathrm{E}$ is present in $63 \%$ of melanomas and up to $50 \%$ of papillary thyroid carcinomas. Wild-type Raf-1 is often hyperactivated in several human solid tumors due to constitutively active upstream oncogenic Ras mutants or overexpression of

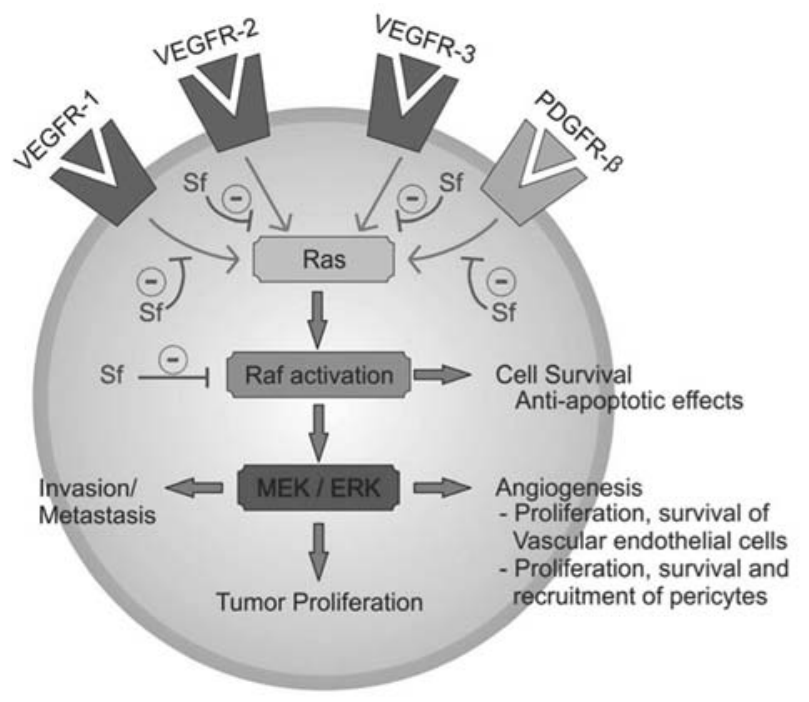

Figure 1. Multiple targets of sorafenib. Sorafenib inhibits auto-phosphorylation of VEGFR-1, VEGFR-2, VEGFR-3 and PDGFR-B, and also prevents Raf activation. The inhibition of Raf is associated with downregulation of downstream signaling and regulation of other pathways that lead to apoptosis.

upstream growth factors or their RTKs. In addition, activated Ras oncogenes such as K-Ras are prevalent in human solid tumors. This includes $90 \%$ of pancreatic, $45 \%$ of colorectal, $30 \%$ HCCs, $35 \%$ non-small cell lung cancers (NSCLC), $15 \%$ of melanomas and 10\% kidney tumors. Also Raf-1 hyperactivation in the absence of oncogenic mutations is commonly seen in RCC and HCC and it is associated with poor prognosis in ovarian and androgen-insensitive prostate cancer (1).

VEGF is expressed in $230-60 \%$ of most solid tumors and in up to $100 \%$ of RCC (8). Overall different tumors have been shown to express different VEGF ligands. For instance VEGFR-1 and VEGFR-2 are upregulated in intratumoral endothelial cells, circulating endothelial cells, endothelial progenitor cells and tumor cells.

As sorafenib acts via various targets, its mechanism of action in different cancers is different depending on the expression of targets. It may be impossible to determine the role of individual targets in cancers but the preclinical data can be used to understand the contribution of various mechanisms of sorafenib in different cancers (5). These mechanisms are summarized in Table I.

\section{Sorafenib monotherapy}

Clinical trials investigating the effect of sorafenib as a single agent have yielded encouraging results in some human solid tumors. Currently sorafenib is approved by the US Food and Drug Administration (FDA) for the treatment of patients with advanced RCC and those with unresectable HCC (9). It is also approved by the European Medicine Agency (EMEA) for the treatment of patients with HCC and advanced RCC in whom previous interferon (IFN)- $\alpha$ or interleukin (IL)-2-based therapy had failed or in those considered to be unsuitable for such therapy.

Renal cell carcinoma. Two randomized controlled clinical trials established the safety and efficacy of sorafenib in 
Table I. Preclinical studies on mechanism of action of sorafenib in different types of cancer.

\begin{tabular}{|c|c|c|}
\hline Cancer type & Study & Findings \\
\hline $\mathrm{RCC}$ & $\begin{array}{l}\text { Effects of sorafenib } \\
\text { on RCC (40) }\end{array}$ & $\begin{array}{l}\text { Sorafenib showed potent tumor growth inhibition and tumor stasis which } \\
\text { strongly correlated with decreased tumor angiogenesis due to inhibition of } \\
\text { PDGF-mediated endothelial and pericyte survival. }\end{array}$ \\
\hline $\mathrm{HCC}$ & $\begin{array}{l}\text { Effects of sorafenib } \\
\text { in HCC cell lines ( } 3 \text { ) }\end{array}$ & $\begin{array}{l}\text { Antitumor activity was observed and was attributed to inhibition of tumor angio- } \\
\text { genesis (VEGFR and PDGFR) and direct effects on tumor cell proliferation/ } \\
\text { survival (Raf kinase signaling-dependent and signaling-independent mechanisms). }\end{array}$ \\
\hline $\begin{array}{l}\text { Breast, } \\
\text { colon } \\
\text { and } \\
\text { NSCLC }\end{array}$ & $\begin{array}{l}\text { In vitro effects of sorafenib } \\
\text { on various kinase residues } \\
\text { and breast cancer, } \\
\text { colon cancer and NSCLC } \\
\text { cell lines (7) }\end{array}$ & $\begin{array}{l}\text { Breast cancer: inhibition of the MAPK pathway and inhibition of angiogenesis. } \\
\text { In the MDA-MB-231 model, sorafenib induced tumor shrinkage, inhibited } \\
\text { proliferation and angiogenesis. Different mechanisms in different colon cancer } \\
\text { models. HT-29: inhibition of both MAPK and angiogenesis pathways; } \\
\text { Colo-205: inhibition of angiogenesis only; A549 NSCLC models: inhibited } \\
\text { Mcl-1 and induced apoptosis-independent of the MAPK pathway. }\end{array}$ \\
\hline
\end{tabular}

Colon In vitro and in vivo blockade cancer of the Raf/ MEK/ERK pathway inhibition by sorafenib in human tumors including the HCT 116 colon tumor model (41)

Melanoma Identified the role of V599E

Inhibition of V599E B-Raf with sorafenib reduced activity of MAPK cascade, B-Raf in melanoma and mechanism of its tumorigenesis (42)

Pancreatic A preclinical study that cancer evaluated the activity of sorafenib on pancreatic cancer cell lines (43)

Sorafenib decreased ERK phosphorylation and inhibited Raf activity in HCT 116 xenografts. inhibited vascular development by decreased VEGF secretion, subsequent increased in tumor cell apoptosis.

Exerted antiproliferative effects independent of RAF/MEK/ERK pathway. Highest levels of apoptosis seen in $\mathrm{t} 3 \mathrm{~m} 4$ cells associated with RAF/AKT/ STAT3 signaling. U0126-induced MEK inhibition did not induce apoptosis in any cell line, reinforcing the hypothesis of a MEK/ERK-independent mechanism by sorafenib. Mcl-1 appears to play an important role in apoptosis. Inhibition of MAPK14 and panAKT phosphorylation.

Lymphoma A preclinical study that analyzed the cytotoxic effect of sorafenib on lymphoma cells (44)

Chronic A preclinical study that Sorafenib-induced cell death of CLL cells accompanied by a loss of Mcl-1. evaluated the mechanism and leukemia activity of sorafenib in chronic lymphocytic leukemia (45)

patients with advanced RCC. The first one was a phase III randomized, double-blind, placebo-controlled TARGET trial with 903 patients who had failed previous standard therapy (10). These patients were randomly assigned to either sorafenib $400 \mathrm{mg}$ twice daily orally or placebo. The primary end-point was overall survival. Crossover from placebo to sorafenib was permitted after a single planned analysis of progression-free survival (PFS), which showed statistically significant benefit over placebo. The median PFS for sorafenib group was 5.5 months while that of placebo group was 2.8 [hazard ratio $(\mathrm{HR})=0.44,95 \%$ confidence interval $(\mathrm{CI}), 0.35-0.55 ; \mathrm{P}<0.01]$. The most common adverse effects were diarrhea, rash, fatigue and hand-foot skin reactions (HFSR). Hypertension and cardiac ischemia were rare serious adverse events, more common in the sorafenib group (10). The final overall survival data showed a statistically significant benefit in the sorafenib group after the post-crossover placebo survival data was censored (17.8 vs. 14.3 months; $\mathrm{HR}=0.78 ; \mathrm{P}=0.029$ ) (9).

The other study was a phase II randomized discontinuation trial, which evaluated the effect of sorafenib on 65 patients who had stable disease after 12 weeks on sorafenib (11). The primary end-point was the percentage of patients remaining progression-free at 24 weeks after sorafenib initiation. At 24 weeks, $50 \%$ of sorafenib-treated patients were progressionfree compared to $18 \%$ of placebo-treated patients $(\mathrm{P}=0.0077)$. Median PFS was significantly longer with sorafenib (24 weeks) 
Table II. Clinical trials of sorafenib monotherapy in various types of cancer.

\begin{tabular}{ll}
\hline Cancer type & \multicolumn{1}{c}{ Study } \\
\hline Non-small & A phase II trial that evaluated the \\
cell lung & $\begin{array}{l}\text { antitumor response and tolerability } \\
\text { of sorafenib in patients with } \\
\text { relapsed or refractory advanced } \\
\text { NSCLC (46) }\end{array}$
\end{tabular}

Review of long-term safety and tolerability of sorafenib in patients with advanced NSCLC (47)

$\begin{array}{ll}\text { Melanoma } & \begin{array}{l}\text { A phase II randomized discontinu } \\ \text { ation trial that evaluated the efficacy } \\ \text { and safety of sorafenib in advanced } \\ \text { melanoma (20) }\end{array} \\ \text { Prostate } & \begin{array}{l}\text { A phase II clinical study that } \\ \text { investigated the activity of } \\ \text { sancer }\end{array} \\ & \begin{array}{l}\text { sorafenib in hormone refractory } \\ \text { prostate cancer (HRPC) (48) }\end{array}\end{array}$

Sarcoma A multicenter phase II study of daily oral sorafenib in patients with recurrent or metastatic sarcoma (49)

Squamous Phase II trial evaluating the efficacy carcinoma and safety of sorafenib in patients of the head with recurrent and/ or metastatic and neck squamous cell carcinoma of the head and neck and nasopharyngeal carcinoma (50)

Biliary tract Phase II trial examining the activity carcinoma of sorafenib in advanced biliary tract carcinoma (51)

Uterine carci- Phase II study of the efficacy and noma and safety of sorafenib in patients with carcino- advanced uterine carcinoma and sarcomas carcinosarcomas (52)
No complete or partial responses observed, stable disease (SD) was achieved in 59\% patients, 4 patients developed tumor cavitation, median PFS was 2.7 months and median OS was 6.7 months, patients with SD had a median PFS of 5.5 months; major grade (3-4) adverse events included hand-foot skin reaction, hypertension, fatigue and diarrhea.

In patients who achieved prolonged response or stable disease with Sorafenib alone or in combination with other drug, sorafenib treatment could be continued until disease progression without major long-term safety or tolerability problems.

Best responses for patients on sorafenib were $19 \%$ stable disease, $62 \%$ progressive disease; overall median PFS was 11 weeks.

Most common adverse effects were dermatological.

Sorafenib was well-tolerated, but had little or no antitumor activity.

Sorafenib showed moderate anticancer activity in HRPC patients, mildtoxicity profile and was well-tolerated, with regard to quality of life; patients had a benefit over conventional chemotherapy.

Sorafenib had activity against angiosarcoma and minimal activity against other sarcomas.

Well-tolerated; modest anticancer activity comparable to monotherapy with other targeted agents in this group of patients.

Showed low activity in cholangiocarcinoma; the toxicity profile was manageable.

Objective response rates were low especially for the more aggressive carcinosarcomas; minimal antitumor activity in advanced uterine carcinoma in some patients. than with placebo ( 6 weeks; $\mathrm{P}=0.087$ ). After this sorafenib was restarted in patients who were on placebo with a median PFS of 24 weeks. Common side effects were skin rash/desquamation, HFSR and fatigue. Although patients on sorafenib experienced more side effects, the overall rate of events was low and the toxic effects were moderate and easily manageable $(10,12)$. Bukowski et al demonstrated that sorafenib shows clinical benefit without adversely impacting overall quality-of-life (QOL) and has a positive impact on some individual symptoms and concerns (13).

Hepatocellular carcinoma. HCC is a highly vascularized, VEGF-driven tumor. No therapeutically beneficial drug in advanced inoperable HCC was available until 2007 (9). It came as a breakthrough discovery in the field when sorafenib improved overall survival (OS) in advanced HCC patients in a phase II randomized placebo-controlled trial (14). Subsequently two phase III randomized double-blind placebocontrolled trials were conducted.

The SHARP trial evaluated 602 patients with advanced HCC who had not received prior systemic therapy, to receive either sorafenib or placebo with the primary endpoint being OS (14). The median OS was significantly longer in the sorafenib group than in the placebo group (10.7 vs. 7.9 months; $\mathrm{P}<0.001)$. The time to radiologic progression was also significantly longer in the sorafenib group compared to placebo 


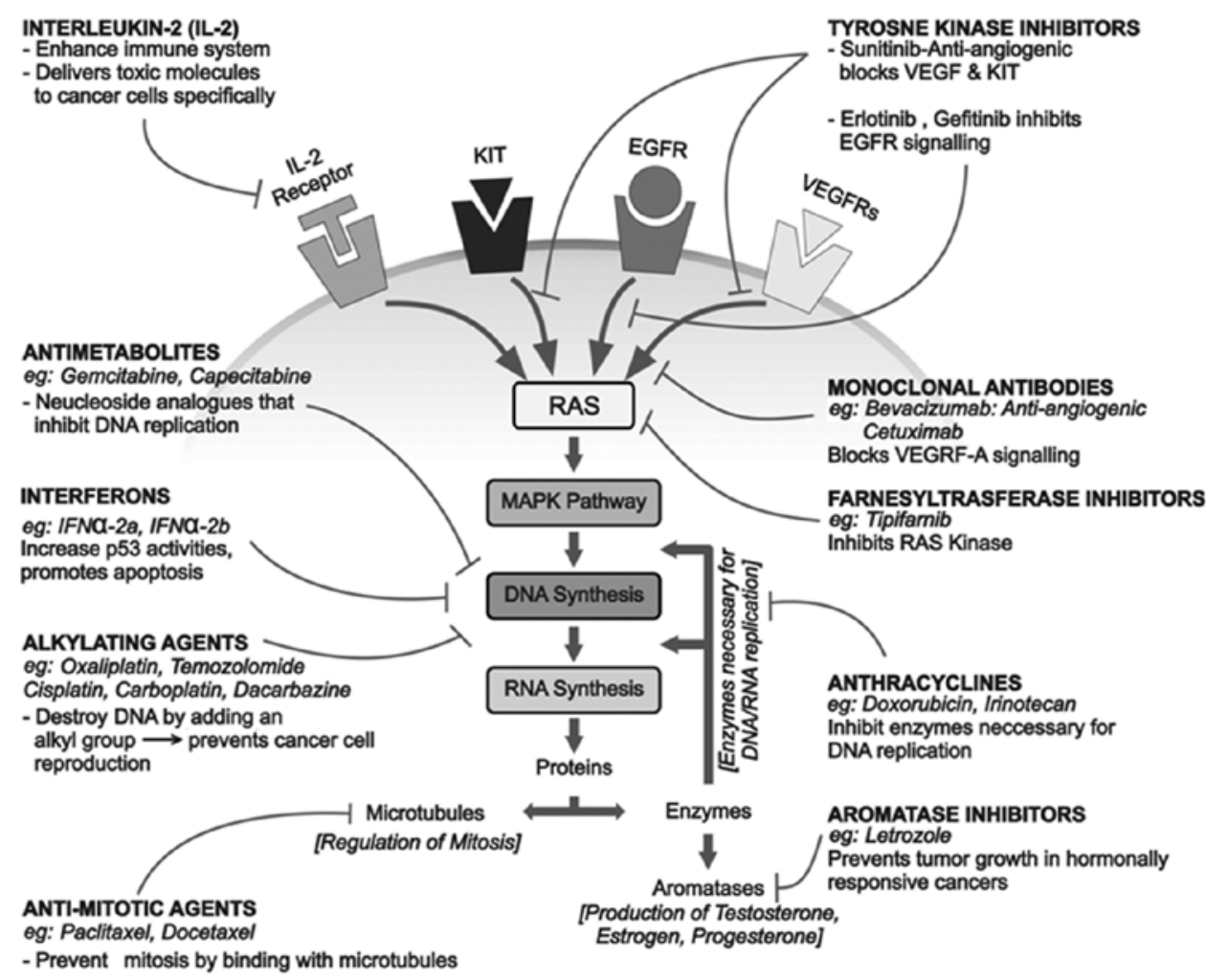

Figure 2. Interaction of various targeted therapeutic agents with cell functioning. Mechanisms of action of chemotherapeutic and molecular targeted agents, which are used in combination with sorafenib.

group (5.5 vs. 2.8 months; $\mathrm{P}<0.001$ ) and the estimated PFS rate at 4 months was 62 and $42 \%$, respectively. Moreover, the disease control was significantly better in the sorafenib group than in the placebo group ( 43 vs. $32 \%, \mathrm{P}=0.002$ ). However, the adverse events were also more frequent in the sorafenib group (80 vs. $52 \%$ ). These effects were mainly of grade 1 or 2 in severity and included diarrhea, weight loss, HFSR, anorexia and alopecia.

These results were affirmed by the results from the AsiaPacific trial, which enrolled patients with similar eligibility criteria. The median OS was 6.5 months in the sorafenib group compared with 4.2 months in the placebo group, while the median time to progression was also significantly prolonged in the sorafenib vs. the placebo group (2.8 vs. 1.4 months; $\mathrm{P}=0.0005)$ (15). The side effect profile was also similar to the SHARP trial.

Although both trials reported an increased adverse event frequency in sorafenib-treated patients, the drug was welltolerated and the side effects were manageable $(15,16)$. The absolute risk of discontinuation or dose reductions was low and could be mitigated by more aggressive monitoring and management.

In order to predict the long-term prognosis after resection of tumors, a preclinical study was undertaken to investigate the effects of sorafenib on tumor growth, recurrence and metastasis after curative resection of HCC in mice (17). This study revealed that sorafenib inhibited the recurrence and metastasis of $\mathrm{HCC}$ after $\mathrm{HCC}$ resection, producing a maximum of $88 \%$ reduction in intrahepatic recurrent tumor volume and a $100 \%$ reduction in lung metastasis. It also showed a 10.7-fold reduction in tumor recurrence. A phase III study to evaluate the use of sorafenib to prevent recurrence and to improve survival after surgical resection or local ablation (STORM study) is currently underway.

Thyroid cancer. Three phase II studies have been conducted to determine the efficacy of sorafenib in advanced follicular thyroid cancer (18). These studies showed that sorafenib had significant activity with partial responses ranging from $15-25 \%$ and stabilization of the disease occurring in an additional $34-56 \%$ of the patients. The median PFS ranged from 13.5 to 19.6 months. Furthermore, the overall safety profile was acceptable and adverse events were consistent with other sorafenib trials (19). In light of these promising results, a phase III study evaluating the efficacy and safety of sorafenib in advanced/metastatic iodine refractory thyroid cancer was initiated (18). Additionally, some guidelines have begun to recommend sorafenib in patients with iodine-refractory progressive metastatic disease who are not willing or able to enter previous clinical trials.

However, in some other cancers like melanoma, sorafenib monotherapy still continues to elude clinical benefit. As mentioned before there is a strong preclinical and clinical evidence supporting the role of B-Raf in driving melanoma progression. In spite of this, sorafenib, being a B-Raf inhibitor, did not demonstrate significant activity in advanced melanoma patients $(20,21)$. Subsequent preclinical studies also showed that sorafenib treatment just led to minor inhibition of B-Raf with many off-target effects (21). Therefore, sorafenib alone did not offer much benefit in melanoma treatment. Similar results were observed in many other types of cancers, of which some are summarized in Table II. 
Table III. Additional studies of sorafenib in combination with chemotherapy or other targeted agents.

\begin{tabular}{ll}
\hline Combination & \multicolumn{1}{c}{ Study } \\
\hline $\begin{array}{l}\text { Additional studies on sorafenib with other targeted agents } \\
\text { Sorafenib + tipifarnib }\end{array}$ & $\begin{array}{l}\text { Evaluated the safety, maximum } \\
\text { tolerated dose, pharmacokinetics } \\
\text { and biological effects of the } \\
\text { combination (53) }\end{array}$ \\
Evafenib + gefitinib & $\begin{array}{l}\text { Evaluated this combination in } \\
\text { advanced NSCLC (54) } \\
\text { Phase II study of the combination } \\
\text { in various types of cancer (55) }\end{array}$ \\
Sorafenib + letrozole & $\begin{array}{l}\text { A preclinical study of the } \\
\text { combination in breast } \\
\text { cancer cells (56) }\end{array}$ \\
Sorafenib + everolimus & $\begin{array}{l}\text { A preclinical study of the effects } \\
\text { of everolimus and sorafenib } \\
\text { in a syngeneic orthotopic } \\
\text { model of hepatocellular } \\
\text { carcinoma (57) }\end{array}$
\end{tabular}

Additional studies on sorafenib and chemotherapy

Sorafenib + dacarbazine

A phase I and phase II study that evaluated this combination as a first line treatment in advanced melanoma (58)

Sorafenib + temozolomide

Evaluated the effect of this combination in advanced melanoma (59)

Sorafenib + irinotecan
Investigated the safety, pharmacokinetics and efficacy of this combination in advanced, refractory solid tumors (60)
This combination was well-tolerated; activity was observed, especially in patients with medullary thyroid cancer.

Sorafenib combined with gefitinib was well tolerated, with promising efficacy in patients with advanced NSCLC.

combination with IL-2 did not demonstrate any improved efficacy over sorafenib alone; improvements appeared in patients receiving higher IL-2 dose.

Combination of letrozole and sorafenib produced a synergistic inhibition of cell proliferation; sorafenib may be effective in preventing the acquisition of resistance towards letrozole.

Combination treatment exerted a stronger antitumoral effect on Morris hepatoma cells than monotherapy.

Manageable toxicity profile; some evidence of antitumor activity; little support for additional studies with this combination in B-Raf mutant disease; further studies may be performed in patients without B-Raf mutation.

This combination was well-tolerated and showed activity in melanoma patients.

Sorafenib $400 \mathrm{mg}$ bid can be combined with irinotecan $125 \mathrm{mg} / \mathrm{m}^{2}$ or $140 \mathrm{mg}$ for the treatment of patients with advanced, refractory solid tumors.

\section{Sorafenib combination therapy}

Sorafenib has various characteristics that suggest it would be a useful combination treatment option for advanced malignancies. The targeting of multiple Raf isoforms and various tyrosine kinase inhibitors may help overcome multidrug resistant genes (2). Also its ability to induce apoptosis in various tumors could complement the cytotoxic effects of standard chemotherapies. Evidence also suggests that inhibition of Raf-1 by sorafenib can resensitize tumor cells to radiation and chemotherapy (2). Moreover, the use of sorafenib in combination with other drugs means that the doses can be reduced and hence it might help alleviate the side effects of the drugs. Owing to these reasons and the limitations of monotherapy, its role in combination with other agents has been widely explored. A figure summarizing the mode of action of combination agents used with sorafenib is shown in Fig. 2.
Sorafenib and other targeted agents. A phase I dose-escalation trial of sorafenib and bevacizumab was performed at below-recommended single-agent doses involving 39 patients with various cancers (22). This combination showed promising clinical activity especially in ovarian cancer. However, the rapidity and frequency of dose reductions indicated an intolerable long-term dosage and the need for alternative dosing schedules. Recently another phase I study reported that intermittent sorafenib dosing with bevacizumab has clinical activity, fewer patients require a sorafenib dose reduction and fewer side effects are observed (23).

The combination of sorafenib with erlotinib was investigated in a phase I trial which reported that the combination was well-tolerated and showed promising activity (24). In a phase II trial for the combination, a higher PFS and OS was seen in the EGFR wild-type and the EGFR FISH-negative patients compared to erlotinib alone (25). However, additional 
studies are needed to confirm the benefit of this combination. Recently, a preclinical study of the combination of sorafenib with erlotinib or cetuximab showed synergistic antitumor activity in both colorectal cancer and NSCLC (26).

A phase I study that investigated sorafenib plus IFN- $\alpha-2 \mathrm{a}$ in advanced RCC and melanoma showed preliminary antitumor activity and the doses were well-tolerated (27). Another study investigating sorafenib with IFN- $\alpha-2 b$ in advanced RCC patients showed substantial activity, but the toxicity exceeded that of either drug alone (28). However, dose reductions and breaks between cycles allowed for long term therapy. In contrast, a more recent phase II study that investigated the combination of sorafenib and pegylated IFN- $\alpha-2 b$ in metastatic melanoma patients showed modest clinical activity and some serious side effects including fatal bleeding complications (29). This may have been due to a different dosing schedule or the use of Peg-IFN- $\alpha-2 b$ instead of conventional IFN- $\alpha$. Additional studies are summarized in Table III.

Sorafenib and chemotherapy. Sorafenib in combination with doxorubicin was studied in a phase I dose escalating study in patients with advanced solid tumors, which showed that the increasing dose did not result in significant toxicity and also promising efficacy results were observed (30). Subsequently, a phase II double-blind study was conducted in patients with advanced HCC (31). The median time to progression, OS and PFS were greater with sorafenib plus doxorubicin compared to doxorubicin alone. The degree to which this improvement represents synergism remains to be defined.

An alternative combination with sorafenib, docetaxel and cisplatin was studied as a phase II study in gastric and gastroesophageal junction adenocarcinoma (32). This study showed encouraging efficacy with a tolerable toxicity and hence further investigations are warranted.

A phase I study with sorafenib, carboplatin and paclitaxel (CP) on advanced NSCLC showed encouraging antitumor activity and manageable adverse effects $(33,34)$. However, in a phase III randomized, double blind trial, no clinical benefit was observed from adding sorafenib to $\mathrm{CP}$ as first-line treatment for NSCLC. Likewise in another phase III trial, the addition of sorafenib to CP did not improve PFS or OS over placebo plus $\mathrm{CP}$ and hence cannot be recommended in the second-line setting for patients with advanced melanoma (35).

A phase II study with sorafenib, gemcitabine and capecitabine (GC) in advanced RCC has also shown encouraging results (36). The PFS and response rates were greater than those observed with sorafenib monotherapy or GC and also the adverse events were manageable. Additional studies are summarized in Table III.

Sorafenib and radiotherapy. The spectrum of kinase inhibition and the toxicity profile of sorafenib increases its potential to synergize with radiation through various mechanisms, such as proliferation inhibition, vascular normalization and interference with intracellular signaling pathways (37). Evidence has suggested that Raf-1 caused radiation resistance through an increased radiation-induced potentially lethal damage repair capacity in HCC cell lines (38). Thus targeting these molecules via sorafenib will re-sensitize these cells to radiation.
Plastaras et al demonstrated that sorafenib did not affect cell survival in vitro, but altered the radiation response in a schedule-dependent manner in vivo, with radiation treatment followed sequentially by sorafenib being associated with the greatest antitumor activity (39).

Similar results were observed in another preclinical study, which investigated the effect of sorafenib plus radiation on colorectal cancer cell lines (37). Sorafenib had little effect on radiation response in vitro but was highly effective in vivo, suggesting that inhibition of proliferation and interference with angiogenesis may be the basis for the interaction. However, there was an interesting observation in the investigation of the timing of sorafenib and radiation through the inhibition assay. A significant growth inhibition was observed when sorafenib was given $2 \mathrm{~h}$ after radiation in the HT29 cell line. This correlates with the results of Plastaras et al, although their conclusion was derived from in vivo studies (39). Following these promising results, studies are currently underway investigating the efficacy in other tumor types as well (37).

\section{Conclusions}

The enhanced understanding of the etiology of cancer has led to an era of molecular targeted therapies that aim to achieve tumor selectivity and limit drug-related toxicities. Sorafenib has emerged as a promising means of addressing these issues with its multiple mechanisms of action and favorable safety and efficacy profile. This resulted in its FDA approval for the treatment of advanced RCC and HCC. However, in many other cancers, it is still limited in activity due to reasons not clearly understood. Fortunately the utility of sorafenib in cancer therapy does not end with this, because its unique and multiple mechanisms of action on tumor and tumor microvasculature has proven valuable when combined with other anticancer therapies, such as other molecular targeted agents, chemotherapy and radiotherapy. A growing body of clinical research supports these combinations and expects that these novelties will soon make their way into cancer treatments. Therefore, sorafenib has not yet failed its original promise. Future issues include identifying optimal combinations, treatment schedules and dosage of sorafenib combinations for a variety of tumor types. In addition, the appropriate biomarkers for improved patient selection and response end-points need to be defined and validated in order to improve the benefits of sorafenib treatment.

\section{References}

1. Wilhelm S, Carter C, Lynch M, et al: Discovery and development of sorafenib: a multikinase inhibitor for treating cancer. Nat Rev Drug Discov 5: 835-844, 2006.

2. Dal Lago L, D'Hondt V and Awada A: Selected combination therapy with sorafenib: a review of clinical data and perspectives in advanced solid tumors. Oncologist 13: 845-858, 2008.

3. Liu L, Cao Y, Chen C, et al: Sorafenib blocks the RAF/MEK/ ERK pathway, inhibits tumor angiogenesis, and induces tumor cell apoptosis in hepatocellular carcinoma model PLC/PRF/5. Cancer Res 66: 11851-11858, 2006.

4. Murdoch D and Sager J: Will targeted therapy hold its promise? An evidence-based review. Curr Opin Oncol 20: 104-111, 2008.

5. Wilhelm SM, Adnane L, Newell P, Villanueva A, Llovet JM and Lynch M: Preclinical overview of sorafenib, a multikinase inhibitor that targets both Raf and VEGF and PDGF receptor tyrosine kinase signaling. Mol Cancer Ther 7: 3129-3140, 2008. 
6. McDermott DF, Sosman JA, Gonzalez R, et al: Double-blind randomized phase II study of the combination of sorafenib and dacarbazine in patients with advanced melanoma: a report from the 11715 Study Group. J Clin Oncol 26: 2178-2185, 2008.

7. Wilhelm SM, Carter C, Tang L, et al: BAY 43-9006 exhibits broad spectrum oral antitumor activity and targets the RAF/ MEK/ERK pathway and receptor tyrosine kinases involved in tumor progression and angiogenesis. Cancer Res 64: 7099-7109, 2004.

8. Longo R and Gasparini G: Challenges for patient selection with VEGF inhibitors. Cancer Chemother Pharmacol 60: 151-170, 2007.

9. Iyer R, Fetterly G, Lugade A and Thanavala Y: Sorafenib: a clinical and pharmacologic review. Exp Opin Pharmacother 11: 1943-1955, 2010

10. Escudier B, Eisen T, Stadler WM, et al: Sorafenib in advanced clear-cell renal-cell carcinoma. N Engl J Med 356: 125-134, 2007.

11. Ratain MJ, Eisen T, Stadler WM, et al: Phase II placebocontrolled randomized discontinuation trial of sorafenib in patients with metastatic renal cell carcinoma. J Clin Oncol 24 2505-2512, 2006.

12. Miyake H, Kurahashi T, Yamanaka K, et al: Impact of sorafenib on health-related quality of life in Japanese patients with metastatic renal cell carcinoma: a prospective evaluation. BJU Int 106: 1643-1647, 2010.

13. Bukowski R, Cella D, Gondek K and Escudier B; Sorafenib TARGETs Clinical Trial Group: Effects of sorafenib on symptoms and quality of life: results from a large randomized placebo-controlled study in renal cancer. Am J Clin Oncol 30 220-227, 2007.

14. Rimassa L and Santoro A: The present and the future landscape of treatment of advanced hepatocellular carcinoma. Dig Liver Dis 42: S273-S280, 2010.

15. Cheng AL, Kang YK, Chen Z, et al: Efficacy and safety of sorafenib in patients in the Asia-Pacific region with advanced hepatocellular carcinoma: a phase III randomised, double-blind, placebo-controlled trial. Lancet Oncol 10: 25-34, 2009.

16. Guan YS and He Q: Sorafenib: activity and clinical application in patients with hepatocellular carcinoma. Exp Opin Pharmacother 12: 303-313, 2011

17. Wang Z, Hu J, Qiu SJ, et al: An investigation of the effect of sorafenib on tumour growth and recurrence after liver cancer resection in nude mice independent of phosphorylated extracellular signal-regulated kinase levels. Exp Opin Invest Drugs 20 1039-1045, 2011.

18. Puxeddu E, Romagnoli S and Dottorini ME: Targeted therapies for advanced thyroid cancer. Curr Opin Oncol 23: 13-21, 2011.

19. Gupta-Abramson V, Troxel AB, Nellore A, et al: Phase II trial of sorafenib in advanced thyroid cancer. J Clin Oncol 26: 4714-4719, 2008

20. Eisen T, Ahmad T, Flaherty KT, et al: Sorafenib in advanced melanoma: a Phase II randomised discontinuation trial analysis. Br J Cancer 95: 581-586, 2006

21. Fedorenko IV, Paraiso KH and Smalley KS: Acquired and intrinsic BRAF inhibitor resistance in BRAF V600E mutant melanoma. Biochem Pharmacol 82: 201-209, 2011.

22. Azad NS, Posadas EM, Kwitkowski VE, et al: Combination targeted therapy with sorafenib and bevacizumab results in enhanced toxicity and antitumor activity. J Clin Oncol 26 : 3709-3714, 2008.

23. Lee JM, Sarosy GA, Annunziata CM, et al: Combination therapy: intermittent sorafenib with bevacizumab yields activity and decreased toxicity. Br J Cancer 102: 495-499, 2010

24. Duran I, Hotté SJ, Hirte H, et al: Phase I targeted combination trial of sorafenib and erlotinib in patients with advanced solid tumors. Clin Cancer Res 13: 4849-4857, 2007.

25. Spigel DR, Burris HA III, Greco FA, et al: Randomized, doubleblind, placebo-controlled, phase II trial of sorafenib and erlotinib or erlotinib alone in previously treated advanced non-small cell lung cancer. J Clin Oncol 29: 2582-2589, 2011.

26. Martinelli E, Troiani T, Morgillo F, et al: Synergistic antitumor activity of sorafenib in combination with epidermal growth factor receptor inhibitors in colorectal and lung cancer cells. Clin Cancer Res 16: 4990-5001, 2010.

27. Escudier B, Lassau N, Angevin E, et al: Phase I trial of sorafenib in combination with IFN $\alpha$-2a in patients with unresectable and/ or metastatic renal cell carcinoma or malignant melanoma. Clin Cancer Res 13: 1801-1809, 2007.
28. Gollob JA, Rathmell WK, Richmond TM, et al: Phase II trial of sorafenib plus interferon $\alpha 2 b$ as first-or second-line therapy in patients with metastatic renal cell cancer. J Clin Oncol 25: 3288-3295, 2007.

29. Egberts F, Gutzmer R, Ugurel S, et al: Sorafenib and pegylated interferon- $\alpha 2 b$ in advanced metastatic melanoma: a multicenter phase II DeCOG trial. Ann Oncol 22: 1667-1674, 2011.

30. Richly H, Henning BF, Kupsch P, et al: Results of a Phase I trial of sorafenib (BAY 43-9006) in combination with doxorubicin in patients with refractory solid tumors. Ann Oncol 17: 866-873, 2006

31. Abou-Alfa GK, Johnson P, Knox JJ, et al: Doxorubicin plus sorafenib vs doxorubicin alone in patients with advanced hepatocellular carcinoma: a randomized trial. JAMA 304: 2154-2160, 2010.

32. Sun W, Powell M, O'Dwyer PJ, Catalano P, Ansari RH and Benson AB III: Phase II study of sorafenib in combination with docetaxel and cisplatin in the treatment of metastatic or advanced gastric and gastroesophageal junction adenocarcinoma: ECOG 5203. J Clin Oncol 28: 2947-2951, 2010.

33. Okamoto I, Miyazaki M, Morinaga R, et al: Phase I clinical and pharmacokinetic study of sorafenib in combination with carboplatin and paclitaxel in patients with advanced non-small cell lung cancer. Invest New Drugs 28: 844-853, 2010.

34. Schiller JH, Flaherty KT, Redlinger M, et al: Sorafenib combined with carboplatin/paclitaxel for advanced non-small cell lung cancer: a phase I subset analysis. J Clin Oncol 24: 7194, 2006.

35. Hauschild A, Agarwala SS, Trefzer U, et al: Results of a phase III, randomized, placebo-controlled study of sorafenib in combination with carboplatin and paclitaxel as second-line treatment in patients with unresectable stage III or stage IV melanoma. J Clin Oncol 27: 2823-2830, 2009.

36. Bellmunt J, Trigo JM, Calvo E, et al: Activity of a multitargeted chemo-switch regimen (sorafenib, gemcitabine, and metronomic capecitabine) in metastatic renal-cell carcinoma: a phase 2 study (SOGUG-02-06). Lancet Oncol 11: 350-357, 2010

37. Suen AW, Galoforo S, Marples B, et al: Sorafenib and radiation: a promising combination in colorectal cancer. Int $\mathrm{J}$ Radiat Oncol Biol Phys 78: 213-220, 2010.

38. Tang WY, Chau SP, Tsang WP, Kong SK and Kwok TT: The role of Raf-1 in radiation resistance of human hepatocellular carcinoma Hep G2 cells. Oncol Rep 12: 1349-1354, 2004.

39. Plastaras JP, Kim SH, Liu YY, et al: Cell cycle-dependent and schedule-dependent antitumor effects of sorafenib combined with radiation. Cancer Res 67: 9443-9454, 2007.

40. Chang YS, Adnane J, Trail PA, et al: Sorafenib (BAY 43-9006) inhibits tumor growth and vascularization and induces tumor apoptosis and hypoxia in RCC xenograft models. Cancer Chemother Pharmacol 59: 561-574, 2007.

41. Wilhelm S and Chien DS: BAY 43-9006: preclinical data. Curr Pharm Des 8: 2255-2257, 2002.

42. Sharma A, Trivedi NR, Zimmerman MA, Tuveson DA, Smith CD and Robertson GP: Mutant V599EB-Raf regulates growth and vascular development of malignant melanoma tumors. Cancer Res 65: 2412-2421, 2005.

43. Ulivi P, Arienti C, Amadori D, et al: Role of RAF/MEK/ERK pathway, p-STAT-3 and Mcl-1 in sorafenib activity in human pancreatic cancer cell lines. J Cell Physiol 220: 214-221, 2009.

44. Chapuy B, Schuelper N, Panse M, et al: Multikinase inhibitor sorafenib exerts cytocidal efficacy against Non-Hodgkin lymphomas associated with inhibition of MAPK14 and AKT phosphorylation. Br J Haematol 152: 401-412, 2011

45. Huber S, Oelsner M, Decker T, et al: Sorafenib induces cell death in chronic lymphocytic leukemia by translational downregulation of Mcl-1. Leukemia 25: 838-847, 2011.

46. Blumenschein GR Jr, Gatzemeier U, Fossella F, et al: Phase II, multicenter, uncontrolled trial of single-agent sorafenib in patients with relapsed or refractory, advanced non-small-cell lung cancer. J Clin Oncol 27: 4274-4280, 2009.

47. Adjei AA, Blumenschein GR Jr, Mandrekar S, Hillman S, Gatzemeier U and Heigener D: Long-term safety and tolerability of sorafenib in patients with advanced non-small cell lung cancer: a case-based review. Clin Lung Cancer 12: 212-217, 2011.

48. Steinbild S, Mross K, Frost A, et al: A clinical phase II study with sorafenib in patients with progressive hormone-refractory prostate cancer: a study of the CESAR Central European Society for Anticancer Drug Research-EWIV. Br J Cancer 97: 1480-1485, 2007.

49. Maki RG, D'Adamo DR, Keohan ML, et al: Phase II study of sorafenib in patients with metastatic or recurrent sarcomas. J Clin Oncol 27: 3133-3140, 2009. 
50. Elser C, Siu LL, Winquist E, et al: Phase II trial of sorafenib in patients with recurrent or metastatic squamous cell carcinoma of the head and neck or nasopharyngeal carcinoma. J Clin Oncol 25: 3766-3773, 2007.

51. Bengala C, Bertolini F, Malavasi N, et al: Sorafenib in patients with advanced biliary tract carcinoma: a phase II trial. Br J Cancer 102: 68-72, 2009.

52. Nimeiri HS, Oza AM, Morgan RJ, et al: A Phase II study of sorafenib in advanced uterine carcinoma/carcinosarcoma: a trial of the Chicago, PMH, and California Phase II Consortia. Gynecol Oncol 117: 37-40, 2010.

53. Hong DS, Sebti SM, Newman RA, et al: Phase I trial of a combination of the multikinase inhibitor sorafenib and the farnesyltransferase inhibitor tipifarnib in advanced malignancies. Clin Cancer Res 15: 7061-7068, 2009.

54. Adjei AA, Molina JR, Mandrekar SJ, et al: Phase I trial of sorafenib in combination with gefitinib in patients with refractory or recurrent non-small cell lung cancer. Clin Cancer Res 13: 2684-2691, 2007.

55. Procopio G, Verzoni E, Bracarda S, et al: Sorafenib with interleukin-2 vs sorafenib alone in metastatic renal cell carcinoma: the ROSORC trial. Br J Cancer 104: 1256-1261, 2011.
56. Bonelli MA, Fumarola C, Alfieri RR, et al: Synergistic activity of letrozole and sorafenib on breast cancer cells. Breast Cancer Res Treat 124: 79-88, 2010

57. Piguet AC, Saar B, Hlushchuk R, et al: Everolimus augments the effects of sorafenib in a syngeneic orthotopic model of hepatocellular carcinoma. Mol Cancer Ther 10: 1007-1017, 2011.

58. Eisen T, Marais R, Affolter A, et al: Sorafenib and dacarbazine as first-line therapy for advanced melanoma: phase I and openlabel phase II studies. Br J Cancer 105: 353-359, 2011.

59. Amaravadi RK, Schuchter LM, McDermott DF, et al: Phase II trial of temozolomide and sorafenib in advanced melanoma patients with or without brain metastases. Clin Cancer Res 15: 7711-7718, 2009.

60. Mross K, Steinbild S, Baas F, et al: Results from an in vitro and a clinical/pharmacological phase I study with the combination irinotecan and sorafenib. Eur J Cancer 43: 55-63, 2007. 